Frascati Physics Series Vol. VVVVVV (xxxx), pp. 000-000

XX Conference - Location, Date-start - Date-end, Year

\title{
Status of Charm Flavor Physics
}

\author{
I. Shipsey \\ Department of Physics, Purdue University, West Lafayette, IN 47907, U.S.A.
}

\begin{abstract}
The role of charm in testing the Standard Model description of quark mixing and $\mathrm{CP}$ violation through measurements of lifetimes, decay constants and semileptonic form factors is reviewed. Together with Lattice QCD, charm has the potential this decade to maximize the sensitivity of the entire flavor physics program to new physics. and pave the way for understanding physics beyond the Standard Model at the LHC in the coming decade. The status of indirect searches for physics beyond the Standard Model through charm mixing, $C P$-violation and rare decays is also reported.
\end{abstract}

\section{Introduction}

Charm plays a dual role in flavor physics. First it provides important supporting measurements for studies of $C P$-violation in $B$ physics. These measurements test QCD technologies such as Lattice QCD, QCD sum rules and 
chiral theory. The first of these theoretical approaches is the most promising for very precise calculations of decay constants and form factors which are the most relevant supporting measurements for $B$ physics. Second, charm provides unique opportunities for indirect searches for physics beyond the SM.

\section{Big Questions in Flavor Physics}

The big questions in quark flavor physics are: (1) "What is the dynamics of flavor?" The gauge forces of the standard model (SM) do not distinguish between fermions in different generations. The electron, muon and tau all have the same electric charge, quarks of different generations have the same color charge. Why generations? Why three? (2) "What is the origin of baryogenesis?" Sakharov gave three criteria, one is $C P$-violation 1). There are only three known examples of $C P$-violation: the Universe, and the beauty and kaon sectors. However, SM $C P$-violation is too small, by many orders of magnitude, to give rise to the baryon asymmetry of the Universe. Additional sources of $C P$-violation are needed. (3) "What is the connection between flavor physics and electroweak symmetry breaking?" Extensions of the SM, for example supersymmetry, contain flavor and $C P$-violating couplings that should show up at some level in flavor physics but precision measurements and precision theory are required to detect the new physics.

\section{Charm in CKM physics}

This is the decade of precision flavor physics. The goal is to over-constrain the CKM matrix with a range of measurements in the quark flavor changing sector of the SM at the per cent level. If inconsistencies are found between, for example, measurements of the sides and angles of the $B_{d}$ unitarity triangle, it will be evidence for new physics. Many experiments will contribute including BaBar and Belle, CDF, D0 at Fermilab, ATLAS, CMS, and LHC-b at the LHC, BESIII, CLEO-c, and experiments studying rare kaon decays.

However, the study of weak interaction phenomena, and the extraction of quark mixing matrix parameters remain limited by our capacity to deal with non-perturbative strong interaction dynamics. Current constraints on the CKM matrix are shown in Fig. 廿(a). The widths of the constraints, except that of $\sin 2 \beta$, are dominated by the error bars on the calculation of hadronic 

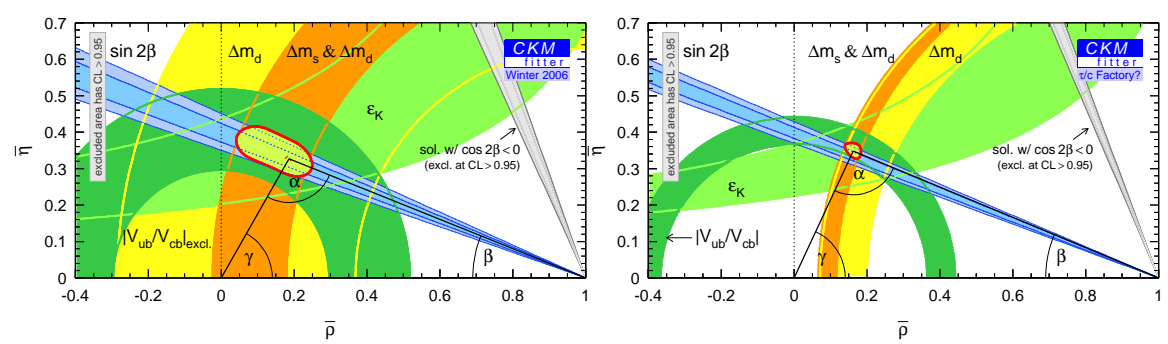

Figure 1: Lattice impact on the $B_{d}$ unitarity triangle from $B_{d}$ and $B_{s}$ mixing, $\left|V_{u b}\right| /\left|V_{c b}\right|, \epsilon_{K}$, and $\sin 2 \beta$. (a) Winter 2006 status of the constraints including the recent observation of $B_{s}$ mixing. (b) Prospects under the assumption that $L Q C D$ calculations of $B$ system decay constants and semileptonic form factors achieve the the precision projected in Table 9.

matrix elements. Recent advances in LQCD have produced calculations of nonperturbative quantities such as $f_{\pi}, f_{K}$, and heavy quarkonia mass splittings that agree with experiment 2). Several per cent precision in charm and beauty decay constants and form factors is hoped for, but the path to higher precision is hampered by the absence of accurate charm data against which to test lattice techniques. This is beginning to change with the BES II run at the $\psi(3770)$, and the start of data taking at the charm and QCD factory CESR-c/CLEO-c 3). Later in the decade BES III at the new double ring accelerator BEPC-II will also turn on 4). CLEO-c is in the process of obtaining charm data samples one to two orders of magnitude larger than any previous experiment, and the BES III data set is expected to be $\sim \times 20$ larger than CLEO-c. These data sets have the potential to provide unique and crucial tests of LQCD, and other QCD technologies such as QCD sum rules and chiral theory, with accuracies of $1-2 \%$.

If LQCD passes the charm factory tests, we will have much greater confidence in lattice calculations of decay constants and semileptonic form factors in $B$ physics. When these calculations are combined with $500 \mathrm{fb}^{-1}$ of $B$ factory data, and improvement in the direct measurement of $\left|V_{t b}\right|$ at the Tevatron 5), they will allow a significant reduction in the size of the errors on $\left|V_{u b}\right|,\left|V_{c b}\right|,\left|V_{t d}\right|$ and $\left|V_{t s}\right|$, quantitatively and qualitatively transforming knowledge of the $B_{d}$ unitarity triangle, see Fig. 1(b), and thereby maximizing the sensitivity of heavy quark physics to new physics. 
Equally important, LQCD combined with charm data allows a significant advance in understanding and control over strongly-coupled, non-perturbative quantum field theories in general. Field theory is generic, weak coupling is not. Two of the three known interactions are strongly coupled: QCD and gravity (string theory). Understanding strongly coupled theories may be a crucial to interpret new phenomena at the high energy frontier.

\subsection{Decay Constants}

The $B_{d}\left(B_{s}\right)$ meson mixing probability can be used to determine $\left|V_{t d}\right|\left(\left|V_{t s}\right|\right)$.

$$
\Delta m_{d} \propto\left|V_{t b} V_{t d}\right|^{2} f_{B_{d}}^{2} B_{B_{d}}
$$

The $B_{d}$ mixing rate is measured with exquisite precision (1\%) 6) but the decay constant is calculated with a precision of about $10-15 \%$. If theoretical precision could be improved to $3 \%$, the error on $\left|V_{t d}\right|$ would be about $5 \%$.

Since LQCD hopes to predict $f_{B} / f_{D^{+}}$with a small error, measuring $f_{D^{+}}$ would allow a precision prediction for $f_{B}$. Hence a precision extraction of $\left|V_{t d}\right|$ from the $B_{d}$ mixing rate becomes possible. Similar considerations apply to $B_{s}$ mixing now it has been observed i.e. a precise determination of $f_{D_{s}^{+}}$would allow a precision prediction for $f_{B_{s}}$ and consequently a precision measurement of $\left|V_{t s}\right|$. Finally the ratio of the two neutral $B$ meson mixing rates determines $\left|V_{t d}\right| /\left|V_{t s}\right|$, but $\left|V_{t s}\right|=\left|V_{c b}\right|$ by unitarity and $\left|V_{c b}\right|$ is known to a few per cent, and so the ratio again determines $V_{t d}$. Which method of determining $\left|V_{t d}\right|$ will have the greater utility depends on which combination of hadronic matrix elements have the smallest error.

Charm leptonic decays measure the charm decay constants $f_{D_{s}^{+}}$and $f_{D^{+}}$ because $\left|V_{c s}\right|$ and $\left|V_{c d}\right|$ are known from unitarity to $0.1 \%$ and $1 \%$ respectively.

$$
\frac{\mathcal{B}\left(D^{+} \rightarrow \mu \nu_{\mu}\right)}{\tau_{D^{+}}}=\text {(const.) } f_{D^{+}}^{2}\left|V_{c d}\right|^{2}
$$

(Charge conjugation is implied throughout this paper.) The measurements are also a precision test of the LQCD. At the start of $2004 f_{D^{+}}$was experimentally undetermined and $f_{D_{s}^{+}}$was known to $33 \%$. 


\subsection{Semileptonic form factors}

$\left|V_{u b}\right|$ is determined from beauty semileptonic decay

$$
\frac{d \Gamma\left(B \rightarrow \pi e^{-} \bar{\nu}_{e}\right)}{d q^{2}}=(\text { const. })\left|V_{u b}\right|^{2} f_{+}^{B \pi}\left(q^{2}\right)^{2}
$$

The differential rate depends on a form factor, $f_{+}\left(q^{2}\right)$ that parameterizes the strong interaction non-perturbative effects. A representative value of $\left|V_{u b}\right|$ determined from $B \rightarrow \pi \ell^{-} \overline{\nu_{e}}$ is 7 ):

$$
\left|V_{u b}\right|=\left(3.76 \pm 0.16_{-0.51}^{+0.87}\right) \times 10^{-3}
$$

where the uncertainties are experimental statistical and systematic, and from the LQCD calculation of the form factor, respectively. The experimental errors are expected to be reduced to $5 \%$ with $B$ factory data samples of $500 \mathrm{fb}^{-1}$ each, and the theory error will dominate.

Again, because the charm CKM matrix elements are known from unitarity, the differential charm semileptonic rate

$$
\frac{d \Gamma\left(D \rightarrow \pi e^{+} \nu_{e}\right)}{d q^{2}}=(\text { const. })\left|V_{c d}\right|^{2} f_{+}^{D \pi}\left(q^{2}\right)^{2}
$$

tests calculations of charm semileptonic form factors. Thus, a precision measurement tests the LQCD calculation of the $D \rightarrow \pi$ form factor. As the form factors governing $B \rightarrow \pi e^{-} \overline{\nu_{e}}$ and $D \rightarrow \pi e^{+} \nu_{e}$ are related by heavy quark symmetry, the charm test gives confidence in the accuracy of the $B \rightarrow \pi$ calculation. The $B$ factories can then use a tested LQCD prediction of the $B \rightarrow \pi$ form factor to extract a precise value of $\left|V_{u b}\right|$. At the start of $2004, \mathcal{B}\left(D \rightarrow \pi e^{+} \nu_{e}\right)$ had been determined to $45 \%[6$, 8 , and the absolute value of the $D \rightarrow \pi$ form factor had not been measured.

Lifetimes of the charm mesons are interpreted within the framework of the Operator Product Expansion. Within OPE the total decay width can be expressed as a series in $1 / m_{c}$ 9).

$$
\begin{array}{r}
\Gamma\left(H_{c}\right)=\Gamma_{\mathrm{c}}+\mathcal{O}\left(1 / m_{c}^{2}\right) \\
+\Gamma_{\mathrm{PI}, \mathrm{WA}, \mathrm{WS}}\left(H_{c}\right)+\mathcal{O}\left(1 / m_{c}^{4}\right)
\end{array}
$$

Mechanisms in which light quarks in the $c$-hadron are involved: Pauli Interference (PI), Weak Annihilation (WA) and Weak Scattering (WS), are $O\left(1 / m_{c}^{3}\right)$ 
Table 1: Charm lifetime world averages in $f s$.

\begin{tabular}{|ll|}
\hline Particle & Lifetime $(\mathrm{fs})$ \\
\hline$D^{+}$ & $1040 \pm 7$ \\
$D_{s}^{+}$ & $504 \pm 4$ \\
$D^{0}$ & $410.3 \pm 1.5$ \\
$\Xi_{c}^{+}$ & $442 \pm 26$ \\
$\Lambda_{c}^{+}$ & $200 \pm 6$ \\
$\Xi_{c}^{+}$ & $112_{-10}^{+13}$ \\
$\Omega_{c}^{+}$ & $69 \pm 12$ \\
\hline
\end{tabular}

but phase space enhanced. The charm lifetimes are in Table 1 The PDG2004 lifetimes are dominated by the exquisitely precise FOCUS measurements from 2002. The $D^{+}$and $D^{0}$ lifetimes are known to 7 and 4 per mille, which is as precise as kaon lifetimes are known. PDG2004 does not include the $D_{s}$ lifetime measurement from FOCUS and so we have averaged it with the PDG value in Table 1 The lifetimes can be explained within OPE 9). To gain a deeper understanding absolute inclusive semileptonic branching ratios of $c$-hadrons, especially the $D_{s}^{+}$and charm baryons, which are currently not well known, need to be measured. For charm CKM physics, the most important point to note is that errors on lifetimes are not a limiting factor in the measurement of absolute rates.

\section{Absolute Charm Branching Ratios}

We reviewed above the importance of absolute charm leptonic and semileptonic branching ratios. The absolute hadronic branching ratios $\mathcal{B}\left(D^{+} \rightarrow K^{-} \pi^{+} \pi^{+}\right)$, $\mathcal{B}\left(D^{0} \rightarrow K^{-} \pi^{+}\right)$, and $\mathcal{B}\left(D_{s}^{+} \rightarrow \phi \pi^{+}\right)$are also important as, currently, all other $D^{+}, D^{0}$ and $D_{s}^{+}$branching ratios are determined from ratios to one or the other of these branching fractions 6). In consequence, nearly all branching fractions in the $B$ and $D$ sectors depend on these reference modes.

Absolute charm branching ratios are poorly known, see Table 2 The reason is that charm produced at $\mathrm{B}$ factories and at the Tevatron or at dedicated fixed target facilities allows relative rate measurements but absolute rate measurements are hard because backgrounds are sizeable, and, crucially, the number of $D$ mesons produced is not easily determined. 
Table 2: Status of important charm branching ratios circa 2004.

\begin{tabular}{|lll|}
\hline Mode & $\mathcal{B}(\%)$ & $\delta \mathcal{B} / \mathcal{B}$ \\
\hline$D^{+} \rightarrow \mu^{+} \nu_{\mu}$ & $0.08_{-0.05}^{+0.17}$ & 100 \\
$D_{s}^{+} \rightarrow \mu^{+} \nu_{\mu}$ & $0.60 \pm 0.14$ & 24 \\
$D^{0} \rightarrow \pi^{-} e^{+} \nu_{e}$ & $0.30_{-0.12}^{+0.23}$ & 45 \\
$D^{0} \rightarrow K^{-} \pi^{+}$ & $3.80 \pm 0.09$ & 2.4 \\
$D^{+} \rightarrow K^{-} \pi^{+} \pi^{+}$ & $9.2 \pm 0.6$ & 6.5 \\
$D_{s}^{+} \rightarrow \phi \pi^{+}$ & $3.6 \pm 0.9$ & 25 \\
$\Lambda_{c}^{+} \rightarrow p K^{-} \pi^{+}$ & $5.0 \pm 1.3$ & 26 \\
$J / \psi \rightarrow \mu^{+} \mu^{-}$ & $5.88 \pm 0.10$ & 1.7 \\
\hline
\end{tabular}

To illustrate one way around this problem consider the clever measurement of $\mathcal{B}\left(D_{s}^{+} \rightarrow \phi \pi^{+}\right)$from the BABAR collaboration 10). The first stage in the analysis is to produce a beam of $D_{s}^{+}$. This is achieved by partially reconstructing $B^{0} \rightarrow D_{s}^{*+} D^{*-}$, where the $D^{*+}$ and the photon in the decay $D_{s}^{*+} \rightarrow D_{s}^{+} \gamma$ are reconstructed but the $D_{s}^{+}$is not observed. BABAR find

$$
\mathcal{B}\left(B^{0} \rightarrow D_{S}^{*+} D^{*-}\right)=(1.88 \pm 0.09 \pm 0.17) \%
$$

In the second step $B^{0} \rightarrow D_{S}^{*+} D^{*-}$ is fully reconstructed

$$
\begin{array}{r}
\mathcal{B}\left(B^{0} \rightarrow D_{s}^{*+} D^{*-}\right) \mathcal{B}\left(D_{s} \rightarrow \phi \pi\right) \\
=(8.81 \pm 0.86) \times 10^{-4}
\end{array}
$$

Dividing these

$$
\mathcal{B}\left(D_{s} \rightarrow \phi \pi\right)=(4.81 \pm 0.52 \pm 0.38) \%
$$

The total error of $12.5 \%$, of which $7.5 \%$ is systematic, represents a dramatic improvement on the $25 \%$ precision of the PDG value. Further improvement in the measurement of this important quantity is expected at the B factories, although it will be challenging to reduce the systematic error significantly. In principle, a several per cent measurement of $\mathcal{B}\left(D_{s} \rightarrow \phi \pi\right)$ is achievable at a charm factory.

\section{BES II and CLEO-c at the $\psi(3770)$}

In 2003 the venerable BES II detector accumulated an integrated luminosity of $33 \mathrm{pb}^{-1}$ at and around the $\psi(3770)$, a factor three greater than the previous 
largest data sample accumulated by Mark III in 1984. The Cornell Electron Storage Ring (CESR) has been upgraded to CESR-c with the installation of 12 wiggler magnets to increase damping at low energies. The CLEO-c detector is a minimal modification of the well understood CLEO III detector. It is the first modern detector to operate at charm threshold. In 2003 a CLEO-c pilot run accumulated $56 \mathrm{pb}^{-1}$ at the $\psi(3770)(360,000 D \bar{D}$ pairs $)$ and this was followed by the first full run accumulating a further $225 \mathrm{pb}^{-1}$ for a total of $281 \mathrm{pb}^{-1}$ at the $\psi(3770)\left(1.8 \times 10^{6} D \bar{D}\right.$ pairs $)$ CLEO-c has also accumulated about $200 \mathrm{pb}^{-1}$ at $\sqrt{s} \sim 4170 \mathrm{MeV}$ for $D_{s}$ physics. These $\psi(3770)$ datasets exceeds those of the BESII (Mark III) experiments by factors of 30 (15). CLEO-c expects to take data until April 2008 and will approximately triple each data set by that time,

In the very near future the BEPCII Project will be commissioned, This is a two ring machine with 93 bunches in each beam. Luminosity is expected to be $10^{33} \mathrm{~cm}^{-2} \mathrm{~s}^{-1}$ at $1.89 \mathrm{GeV} 6 \times 10^{32} \mathrm{~cm}^{-2} \mathrm{~s}^{-1}$ at $1.55 \mathrm{GeV}$ and $6 \times 10^{32} \mathrm{~cm}^{-2} \mathrm{~s}^{-1}$ at $2.1 \mathrm{GeV}$. The linac was installed in 2005. The ring is to be installed this year (2006) and the BESIII detector will be in place and commissioned in 2007 with data taking beginning of 2008 , with early running at the $J / p s i$. Although the detailed run plan has not been decided: an example is given here. At $5 / \mathrm{fb} / \mathrm{yr}$ or $15 / \mathrm{fb} / 3 \mathrm{yrs}$, there will be $90 \times 10^{6} D \bar{D}$ pairs or a factor 20 greater than the full CLEO-c data sample. Three years at $4170 \mathrm{MeV}$ would produce $2 \times 10^{6} D_{s} \bar{D}_{s}$ pairs in three years again a factor 20 greater than the full CLEO-c data set.

In the longer term proposed Super B Factories at KEK or SuperB or a dedicated charm factory would produce an abundance of charm. For example the SuperB machine at $10^{36} \mathrm{~cm}^{-2} \mathrm{~s}^{-1}$ will produce $10^{10} e^{+} e^{-} \rightarrow c \bar{c}$ pairs $/ 10^{7} \mathrm{~s}$. Due to the "Linear Collider design" there is an option to lower the energy to $4 \mathrm{GeV}$ with a modest luminosity penalty of a factor 10 . In this mode of operation the super B Factory becomes a super flavour factory. When discussing charm factory results from CLEO-c I will extrapolate to BEPCII/BESIII (my estimates, not official ones) and to super flavour. For the latter I will assume $1 \times 10^{35}$ for $10^{7} \mathrm{~s}$ which is $\left(6.4 \times 10^{9} \mathrm{D} \bar{D}\right.$ pairs $)$ at the $\psi(3770)$ exceeding the BEPCII and CESR-c data samples by a factor of 70 and 1,000 respectively. 


\subsection{Analysis Technique}

There are decisive advantages to running at charm threshold. As $\psi \rightarrow D \bar{D}$, the technique is to fully reconstruct one $D$ meson in a hadronic final state, the tag, and then to analyze the decay of the second $D$ meson in the event to extract inclusive or exclusive properties.

As $E_{\text {beam }}=E_{D}$, the candidate is required to have energy close to the beam energy, and the beam-constrained candidate mass, $M_{D}=\sqrt{E_{\text {beam }}^{2}-p_{\text {cand }}^{2}}$, is computed. Charm mesons have many large branching ratios to low multiplicity final states, and so the tagging efficiency is very high, about $25 \%$, compared to much less than $1 \%$ for $B$ tagging at a $B$ factory.

Tagging creates a single $D$ meson beam of known momentum. The beam constrained mass for events in which the second $D$ meson is also reconstructed are shown in Fig. 2. These double tag events, which are key to making absolute branching fraction measurements, are pristine. The absolute branching fraction is given by:

$$
\mathcal{B}\left(D^{+} \rightarrow K^{-} \pi^{+} \pi^{+}\right)=\frac{N\left(K^{-} \pi^{+} \pi^{+}\right)}{\epsilon\left(K^{-} \pi^{+} \pi^{+}\right) \times N\left(D^{-}\right)}
$$

where $N\left(K^{-} \pi^{+} \pi^{+}\right)$is the number of $D^{+} \rightarrow K^{-} \pi^{+} \pi^{+}$observed in tagged events, $\epsilon\left(K^{-} \pi^{+} \pi^{+}\right)$is the reconstruction efficiency and $N\left(D^{-}\right)$is the number of tagged events.

In a method similar to that pioneered by Mark III 11, 12), CLEO fits to the observed single tag and double tag yields for six $D^{+}$and three $D^{0}$ modes 13). I will only consider the two most important branching fractions here. For $D^{0} \rightarrow K^{-} \pi^{+}$the total errors are comparable to previous measurements, see Table 3 But the true improvement is that the previous most precise measurements from ALEPH 14) and CLEO 15) were based on comparing $D^{*+} \rightarrow D^{0} \pi_{s}, D^{0} \rightarrow K^{-} \pi^{+}$with and without explicitly reconstructing the $D^{0}$. The latter measurement relies on a correlation between the momentum of the slow pion from the $D^{*}$ and the thrust axis of the $e^{+} e^{-} \rightarrow q \bar{q}$ event. Consequently, these early measurements had poor signal to noise whereas the CLEO-c measurement has a signal to noise of about $60 / 1$.

This is the most precise measurement of $\mathcal{B}\left(D^{+} \rightarrow K^{-} \pi^{+} \pi^{+}\right)$to date, see Table 4 but the improvement is again much more than statistics. The previous most precise measurement, which was from CLEO 16), bootstrapped 


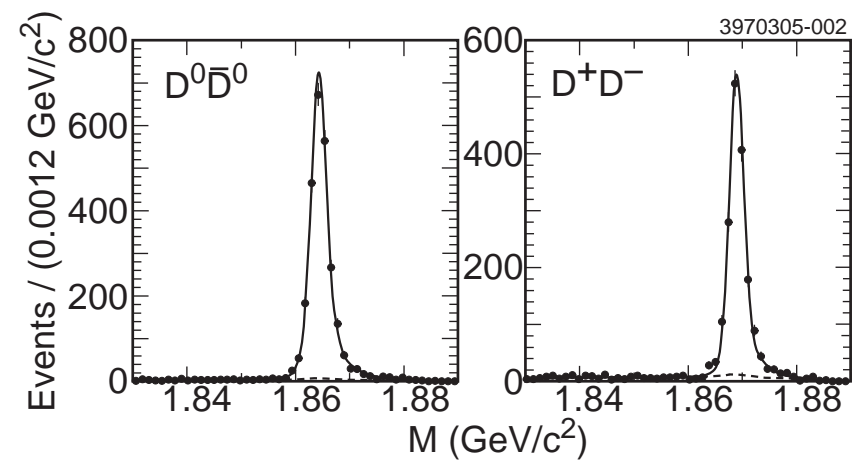

Figure 2: Beam constrained mass of D mesons in CLEO-c events in which both $D$ mesons have been fully reconstructed.

Table 3: The $D^{0} \rightarrow K^{-} \pi^{+}$absolute charm branching ratio.

\begin{tabular}{ll}
\hline $\mathcal{B}(\%)$ & Error (Source) \\
\hline $3.82 \pm 0.07 \pm 0.12$ & $3.6 \%($ CLEO 15) \\
$3.90 \pm 0.09 \pm 0.12$ & $3.8 \%($ ALEPH 14) \\
$3.80 \pm 0.09$ & $2.4 \%($ PDG) \\
$3.91 \pm 0.08 \pm 0.09$ & $3.1 \%($ CLEO-c 13) \\
\hline
\end{tabular}

on $D^{0} \rightarrow K^{-} \pi^{+}$through a measurement of

$$
\frac{\mathcal{B}\left(D^{*+} \rightarrow D^{0} \pi^{+}\right) \mathcal{B}\left(D^{0} \rightarrow K^{-} \pi^{+}\right)}{\mathcal{B}\left(D^{* 0} \rightarrow D^{+} \pi^{0}\right) \mathcal{B}\left(D^{+} \rightarrow K^{-} \pi^{+} \pi^{+}\right)}
$$

so it was not independent of $\mathcal{B}\left(D^{0} \rightarrow K^{-} \pi^{+}\right)$, while the new measurement has no dependence on $\mathcal{B}\left(D^{0} \rightarrow K^{-} \pi^{+}\right)$a much more satisfactory situation.

BES II has performed a similar analysis. These recent measurements are in remarkably good agreement with the PDG averages, indicating that the charm, and hence beauty, decay scales, are approximately correct and are now, finally, on a solid foundation.

The CLEO-c $\psi(3770)$ integrated luminosity goal of $0.75 \mathrm{fb}^{-1}$ may sound small compared to the more than $500 \mathrm{fb}^{-1}$ collected by Belle, and the slightly 
Table 4: The $D^{+} \rightarrow K^{-} \pi^{+} \pi^{+}$absolute charm branching ratio.

\begin{tabular}{ll}
\hline $\mathcal{B}(\%)$ & Error (Source) \\
\hline $9.3 \pm 0.6 \pm 0.8$ & $10.8 \%$ (CLEO 16) \\
$9.1 \pm 1.3 \pm 0.4$ & $14.9 \%$ (MKIII 17) $)$ \\
$9.1 \pm 0.7$ & $7.7 \%$ (PDG) \\
$9.52 \pm 0.25 \pm 0.27$ & $3.9 \%$ (CLEO-c 13) \\
\hline
\end{tabular}

Table 5: Charm factory hadronic branching ratio measurement expected precision with $0.75 \mathrm{fb}^{-1}$ data samples at the $\psi(3770)$ and above $D_{s} \bar{D}_{s}$ threshold. The first uncertainty is statistical and the second systematic.

\begin{tabular}{lll}
\hline Mode & \multicolumn{2}{c}{$\delta \mathcal{B} / \mathcal{B}(\%)$} \\
& PDG2004 & $0.75 \mathrm{fb}^{-1}$ \\
\hline$D^{0} \rightarrow K^{-} \pi^{+}$ & $2.4 \%$ & $0.6 \%, 1.1 \%$ \\
$D^{+} \rightarrow K^{-} \pi^{+} \pi^{+}$ & $7.7 \%$ & $0.7 \%, 1.2 \%$ \\
$D_{s}^{+} \rightarrow \phi \pi$ & $12.5 \%$ 10) & $4.0 \%$ \\
\hline
\end{tabular}

smaller sample by BABAR. However, the ability to perform a tagged analysis is comparable at the two types of factory because the tagging efficiency is at least 25 times larger at a charm factory than at a $B$ factory, and the cross section is about six times larger. Hence,

$$
\frac{N(B \text { tags at a } B \text { factory })}{N(D \text { tags at a charm factory })} \sim 1 .
$$

In consequence the number of events in $100 \mathrm{pb}^{-1}$ with two $D$ mesons reconstructed is about the same as the number of events at $10 \mathrm{GeV}$ with $500 \mathrm{fb}^{-1}$ with two $B$ mesons reconstructed. Projections for the expected precision with which the reference hadronic branching ratios will be measured with a $0.75 \mathrm{fb}^{-1}$ data set are given in Table 5 CLEO-c and, later BES III, will set the scale for all heavy quark measurements. 


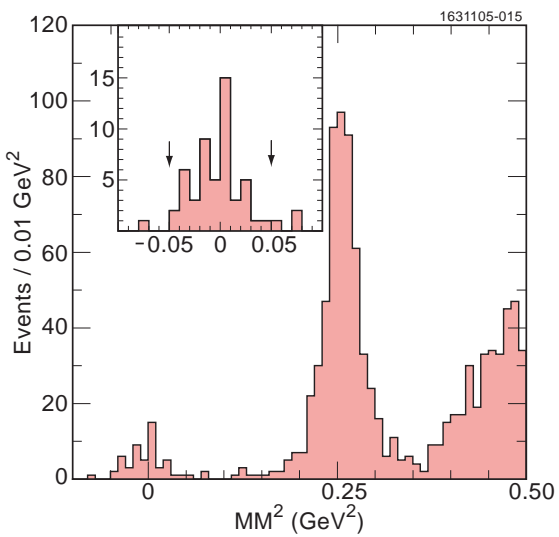

Figure 3: The $M M^{2}$ distribution in events with $D^{-}$tag, a single charged track of the correct sign, and no additional (energetic) showers. The insert shows the signal region for $D^{+} \rightarrow \mu \nu_{\mu}$. A $\pm 2 \sigma$ range is indicated by the arrows.

\subsection{Charm Decay Constant}

The measurement of the leptonic decay $D^{+} \rightarrow \mu^{+} \nu_{\mu}$ benefits from the fully tagged $D^{-}$at the $\psi(3770)$. One observes a single charged track recoiling against the tag that is consistent with a muon of the correct sign. Energetic electromagnetic showers un-associated with the tag are not allowed. The missing mass $M M^{2}=m_{\nu}^{2}$ is computed; it peaks at zero for a decay where only a neutrino is unobserved. Fig. 3 shows the $M M^{2}$ distribution from CLEO-c 18).

There are 50 candidate signal events, and $2.81 \pm 0.3_{-0.22}^{+0.84}$ background events. After correcting for efficiency, CLEO-c finds

$$
\mathcal{B}\left(D^{+} \rightarrow \mu^{+} \nu_{\mu}\right)=\left(4.40 \pm 0.66_{-0.12}^{+0.09}\right) \times 10^{-4},
$$

where the uncertainties are statistical and systematic, respectively. Under the assumption of three generation unitarity, and using the precisely known $D^{+}$ lifetime, CLEO-c obtains

$$
f_{D^{+}}=\left(222.6 \pm 16.7_{-3.4}^{+2.8}\right) \mathrm{MeV} .
$$

This is the most precise measurement of $f_{D^{+}}$18). The result appeared at Lepton-Photon 2005 just two days after the first unquenched lattice QCD cal- 


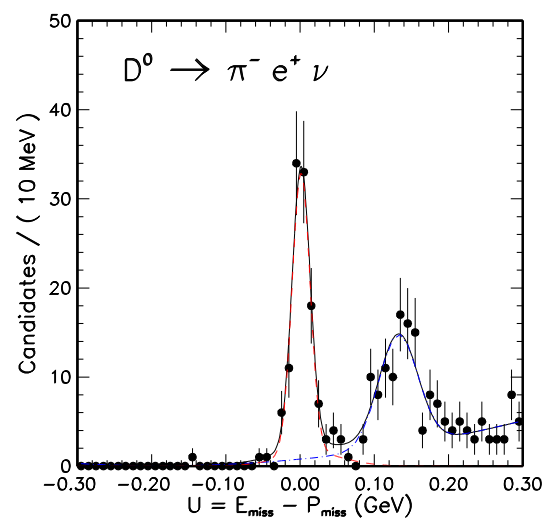

Figure 4: The $U=E_{\text {miss }}-P_{\text {miss }}$ distribution in events with a $\bar{D}^{0}$ tag, a positron, and a single charged track of the correct sign. The peaks at zero and $0.13 \mathrm{GeV}$ correspond to $D^{0} \rightarrow \pi^{-} e^{+} \nu_{e}$ and $D^{0} \rightarrow K^{-} e^{+} \nu_{e}$ (preliminary.)

culation 19) had predicted:

$$
f_{D^{+}}=(201 \pm 3 \pm 17) \mathrm{MeV}
$$

The combined experimental error is $8 \%$ while the LQCD error is also $8 \%$ 19). The results are in good agreement but errors are still large. The only other positive observation of this decay is by BES II who found three candidate events with a background of 0.25 events in their $33 \mathrm{pb}^{-1}$ data sample. They find a branching ratio of $\left(0.122_{-0.053}^{+0.111} \pm 0.010\right) \%$ corresponding to $f_{D^{+}}=\left(371_{-119}^{+129} \pm\right.$ $25) \mathrm{MeV} 20$. The CLEO value is considerably smaller and in better agreement with expectations from the lattice and other theoretical approaches. With $0.75 \mathrm{fb}^{-1}$ a $4.5 \%$ error for $f_{D^{+}}$is expected. Similar precision is expected for $f_{D_{s}^{+}}$at $\sqrt{s}=4160 \mathrm{MeV}$. BES III will make even more precise measurements achieving a precision of several per cent for both $f_{D^{+}}$and $f_{D_{s}^{+}}$which is well matched to the ultimate precision of the LQCD calculations.

\subsection{Measurement of the Charm Semileptonic Form Factors}

The measurement of semileptonic decay absolute branching ratios and absolute form factors is also based on the use of tagged events. The analysis procedure, 
Table 6: Selected CLEO-c charm semileptonic branching ratio measurements in $\%$ and a comparison to the PDG.

\begin{tabular}{lll}
\hline Mode & PDG & CLEO-c \\
\hline$D^{0} \rightarrow \pi^{-} e^{+} \nu_{e}$ & $0.36 \pm 0.06$ & $0.26 \pm 0.03 \pm 0.1$ \\
$D^{0} \rightarrow K^{-} e^{+} \nu_{e}$ & $3.58 \pm 0.18$ & $3.44 \pm 0.10 \pm 0.1$ \\
$D^{+} \rightarrow \pi^{0} e^{+} \nu_{e}$ & $0.31 \pm 0.05$ & $0.44 \pm 0.06 \pm 0.01$ \\
$D^{+} \rightarrow \bar{K}^{0} e^{+} \nu_{e}$ & $6.7 \pm 0.9$ & $8.71 \pm 0.38 \pm 0.37$ \\
\hline
\end{tabular}

using $D^{0} \rightarrow \pi^{-} e^{+} \nu_{e}$ as an example is as follows. A positron and a hadronic track are identified recoiling against the tag. The quantity $U=E_{\text {miss }}-P_{\text {miss }}$ is calculated, where $E_{\text {miss }}$ and $P_{\text {miss }}$ are the missing energy and missing momentum in the event. For a tagged event with a semileptonic decay $E_{m i s s}$ and $P_{\text {miss }}$ are the components of the four-momentum of the neutrino. $U$ peaks at zero if only a neutrino is missing. The $U$ distribution in $56 \mathrm{pb}^{-1}$ of CLEO-c data is shown in Fig. 4 where a clean signal of about 100 events is observed for $D \rightarrow \pi e^{+} \nu_{e}$ with $S / N 20 / 1$ 21). In previous analyses at $B$ Factories and fixed target experiments the background was usally larger than the signal see for example 22).

The kinematic power of running at threshold also allows previously unobserved modes such as $D^{0} \rightarrow \rho^{-} e^{+} \nu_{e}$ to be easily identified 21). BES II have performed similar analyses 23) 24) and results are in good agreement with CLEO-c. Selected CLEO-c absolute semileptonic branching ratio measurements are compared to PDG values in Table 6 .

This modest data sample has already produced several important measurements. The ratio of $\Gamma\left(D^{0} \rightarrow K^{-} e^{+} \nu_{e}\right) / \Gamma\left(D^{+} \rightarrow \bar{K}^{0} e^{+} \nu_{e}\right)$ is expected to be unity by isospin. The PDG value is $1.35 \pm 0.19$ (6) . Using the measured branching fractions for the decays of $D^{0} \rightarrow K^{-} e^{+} \nu_{e}$ and $D^{+} \rightarrow \bar{K}^{0} e^{+} \nu_{e}$ and the lifetimes of the $D^{0}$ and $D^{+}$[6) CLEO-c obtains the ratio of the decay widths

$$
\frac{\Gamma\left(D^{0} \rightarrow K^{-} e^{+} \nu_{e}\right)}{\Gamma\left(D^{+} \rightarrow \bar{K}^{0} e^{+} \nu_{e}\right)}=1.00 \pm 0.05 \pm 0.04
$$

where the first error is statistical and the second systematic. The CLEO0-c result, and a less precise result from BES II, are consistent with unity thereby 


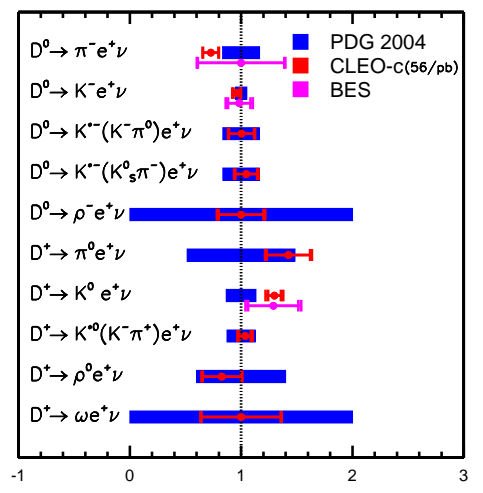

Figure 5: CLEO-c, BES II and the PDG values for a range of charm meson semileptonic branching ratios. Results are normalized to PDG values for ease of display.

solving a long standing puzzle.

As the charm CKM matrix elements are known from unitarity, the absolute differential charm semileptonic rate

$$
\frac{d \Gamma\left(D \rightarrow \pi e^{+} \nu_{e}\right)}{d q^{2}}=(\text { const. })\left|V_{c d}\right|^{2} f_{+}\left(q^{2}\right)^{2}
$$

tests calculations of charm semileptonic form factor $q^{2}$ dependence and form factor magnitude. A precision absolute branching fraction measurement also tests the magnitude of the form factor if an assumption is made about the functional form of the $q^{2}$ dependence. Recently there have been several beautiful measurements of the form factor shape in $D \rightarrow K \ell^{+} \nu_{\ell}$ and $D \rightarrow \pi \ell^{+} \nu_{\ell}$ by CLEO, FOCUS, Belle, and BABAR. By reconstructing two $D$ mesons in $e^{+} e^{-} \rightarrow c \bar{c}$ events at $10 \mathrm{GeV}$ Belle are able to make an absolute measurement and so a determination of the form factor magnitude as well. CLEO-c promise results soon.

In a pseudoscalar to pseudoscalar transition the differential rate is proportional to the third power of the daughter hadron momentum due to the $\mathrm{P}$-wave nature of the decay. The $p^{3}$ term dominates the differential rate. The form factor parameterizes the additional $q^{2}$ dependence of the semileptonic amplitude arising from non-perturbative QCD. The form factor is largest at 
$q^{2}=q_{\max }^{2}$ where the daughter hadron is stationary in the rest frame of the $D$ meson and decreases by about a factor of two at $q^{2}=0$. Since most of the rate is at $q^{2}=0$ it is traditional to normalize the form factor at $q^{2}=0$, however it is simpler to calculate the form factor at $q^{2}=q_{\max }^{2}$ where the rate vanishes as we at the edge of phase space.

Several choices for the functional form of $f_{+}\left(q^{2}\right)$ have been proposed. The simple pole model is a form predicted by vector meson dominance also called nearest pole dominance 25), in which exchange is dominated by the lowest lying vector meson (the spectroscopic pole) with the quantum numbers of the $c \rightarrow s$ transition.

$$
f_{+}\left(q^{2}\right)=\frac{f_{+}\left(q^{2}=0\right)}{1-\frac{q^{2}}{m_{\text {pole }}^{2}}}
$$

Where $m_{\text {pole }}=M_{D^{*}}$ for $D \rightarrow \pi \ell \nu_{\ell}$ and $m_{\text {pole }}=M_{D_{s}^{*}}$ for $D \rightarrow K \ell \nu_{\ell}$. At lower values of $q^{2}$ the spectrum has contributions from higher poles, and to account for this the modified pole or BK parametrization was proposed 26)

$$
f_{+}\left(q^{2}\right)=\frac{f_{+}\left(q^{2}=0\right)}{\left(1-\frac{q^{2}}{m_{\text {pole }}^{2}}\right)\left(1-\frac{\alpha q^{2}}{m_{\text {pole }}^{2}}\right)}
$$

Here $\alpha$ parameterizes the contributions of all additional poles combined, and $m_{\text {pole }}$ remains the spectroscopic pole. The $q^{2}$ spectrum in $D \rightarrow K \ell \nu_{\ell}$ can be described by the pole model within experimental resolution, but the pole mass needed to do so is far from the spectroscopic pole. The B-K parametrization describes the data for $D \rightarrow K \ell \nu_{\ell}$ within the experimental precision and also provides a way to parameterize the lattice calculations. A comparison of a lattice prediction for $\alpha$ to data is shown for FOCUS and BABAR data in Figure [6] The precision of the prediction and the measurements are at the $10 \%$ level. Agreement is good, although the errors are still large.

The FOCUS, BABAR and Belle measurements check the shape of the form factor. The normalization can be checked by either fitting to the differential rate to obtain $f^{+}(0) V_{c x}$ or from the absolute branching fraction, in both cases using unitarity and the $D$ meson lifetime. A comparison of absolute branching fraction measurements and the LQCD prediction is shown in Figure 7 Here while the measurement has recently become much more precise, the precision of the prediction lags experiment significantly. Agreement is reasonable, although the theory errors are in urgent need of being reduced. 


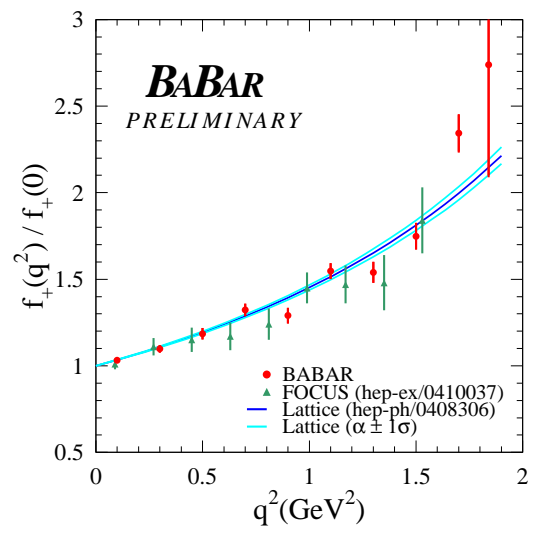

Figure 6: The differential rate, normalized to the rate at $q^{2}=0$ for the decay $D^{0} \rightarrow K^{-} \ell \nu_{\ell}$ after removal of phase space factors, compared to the $L Q C D$ prediction.

Table 7: Experimental measurements and theoretical predictions of shape parameters in $D \rightarrow K$ semileptonic decay.

\begin{tabular}{lll}
\hline Measurement & $\alpha$ & $m_{\text {pole } \mathrm{GeV}}$ \\
\hline E691 1989 27 & - & $2.1_{-0.2}^{+0.4} \pm 0.2$ \\
CLEO 1991 28) & - & $2.0_{-0.2-0.2}^{+0.4+0.3}$ \\
MARKIII 1991 29) & - & $1.8_{-0.2-0.2}^{+0.5+0.3}$ \\
CLEOII 1993 [30) & - & $2.00 \pm 0.12 \pm 0.18$ \\
E687 1995 31) & - & $1.87_{-0.08-0.06}^{+0.11+0.07}$ \\
CLEOIII 2005 22) & $0.36 \pm 0.10_{-0.07}^{+0.03}$ & $1.89 \pm 0.05_{-0.03}^{+0.04}$ \\
FOCUS 2005 [32) & $0.28 \pm 0.08 \pm 0.07$ & $1.93 \pm 0.05 \pm 0.03$ \\
Belle 2006 33) & $0.52 \pm 0.08 \pm 0.06$ & - \\
BABAR 2006 34) & $0.43 \pm 0.03 \pm 0.04$ & $1.854 \pm 0.016 \pm 0.020$ \\
LQCD [35) & $0.50 \pm 0.06 \pm 0.07$ & - \\
LCSR 36) & $-0.07_{-0.07}^{+0.15}$ & - \\
CQM [37) & 0.24 & - \\
\hline
\end{tabular}




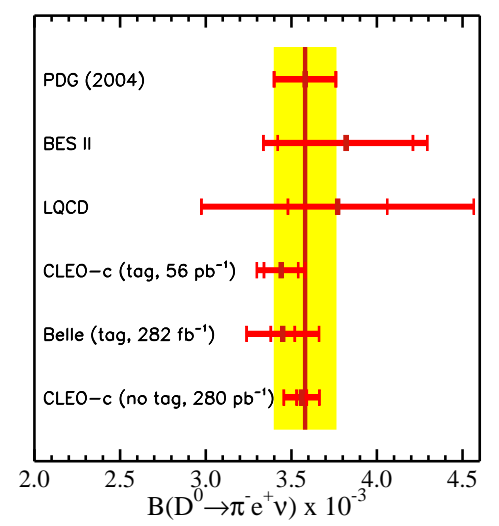

Figure 7: Measurements of the absolute branching fraction for $D \rightarrow \pi e^{+} \nu_{e}$ and comparison to $L Q C D$. A preliminary result from an untagged measurement from CLEO-c has also been included.

The $q^{2}$ resolution at a charm factory is about $0.025 \mathrm{GeV}^{2}$, which is more than a factor of 10 better than CLEO III which achieved a resolution of 0.4 $\mathrm{GeV}^{2}$ 22). This huge improvement is due to the kinematics at the $\psi(3770)$ resonance, i.e. that the $D$ meson momentum is known. (Belle have recently achieved similar $q^{2}$ resolution by using a charm tagging technique at $10 \mathrm{GeV}$.) The combination of large statistics, and excellent kinematics will enable the absolute magnitudes and shapes of the form factors in every charm semileptonic decay to be measured, in many cases to a precision of a few per cent. This is a stringent test of LQCD.

By taking ratios of semileptonic and leptonic rates, CKM factors can be eliminated. Two such ratios are

$$
\begin{array}{r}
\Gamma\left(D^{+} \rightarrow \pi^{0} e^{+} \nu_{e}\right) / \Gamma\left(D^{+} \rightarrow \mu \nu_{\mu}\right) \\
\Gamma\left(D_{s}^{+} \rightarrow(\eta \text { or } \phi) e^{+} \nu_{e}\right) / \Gamma\left(D_{s}^{+} \rightarrow \mu \nu_{\mu}\right)
\end{array}
$$

These ratios depend purely on hadronic matrix elements and can be determined to $8 \%$ and so will test amplitudes at the $4 \%$ level. This is an exceptionally stringent test of LQCD.

If LQCD passes the experimental tests outlined above it will be possible to use the LQCD calculation of the $B \rightarrow \pi$ form factor with increased confidence 
Table 8: Experimental measurements and theoretical predictions of shape parameters in $D \rightarrow \pi$ semileptonic decay.

\begin{tabular}{lll}
\hline Measurement & $\alpha$ & $m_{\text {pole }} \mathrm{GeV}$ \\
\hline CLEOIII 2005 22) & $0.37_{-0.31}^{+0.20} \pm 0.15$ & $1.86_{-0.09-0.03}^{+0.10+0.10}$ \\
FOCUS 2005 32) & - & $1.91_{-0.15}^{+0.03} \pm 0.07$ \\
Belle 2006 33) & $0.10 \pm 0.21 \pm 0.10$ & - \\
LQCD [35) & $0.44 \pm 0.06 \pm 0.07$ & - \\
LCSR 36]) & $0.01_{-0.07}^{+0.11}$ & - \\
CQM 37) & 0.30 & - \\
\hline
\end{tabular}

at the $B$ factories to extract a precision $V_{u b}$ from $B \rightarrow \pi e^{-} \bar{\nu}_{e}$. BaBar and Belle will also be able to compare the LQCD prediction of the shape of the $B \rightarrow \pi$ form factor to data as an additional cross check.

Successfully passing the experimental tests allows the charm factories to use LQCD calculations of the charm semileptonic form factors to directly measure $\left|V_{c d}\right|$ and $\left|V_{c s}\right|$. Using the isospin averaged semileptonic widths $\Gamma(D \rightarrow$ $\left.K e^{+} \nu_{e}\right)$ and $\Gamma\left(D \rightarrow \pi e^{+} \nu_{e}\right)$ 21) and the LQCD prediction of the semileptonic partial width 35 I obtain

$$
\begin{aligned}
& V_{c s}=0.957 \pm 0.017 \pm 0.093 \\
& V_{c d}=0.213 \pm 0.008 \pm 0.029
\end{aligned}
$$

where the uncertainties are experimental statistical, experimental systematic and from LQCD. The results are consistent with the unitarity values

$$
\begin{gathered}
V_{c s}=0.9745 \pm 0.0008 \\
V_{c d}=0.2238 \pm 0.012
\end{gathered}
$$

$V_{c d}$ has previously been determined from neutrino production of di-muons off of nucleons, and $V_{c s}$ has been determined from $W \rightarrow c s$ transitions at LEP to be 6 )

$$
\begin{aligned}
& \left|V_{c s}\right|=0.976 \pm 0.014 \\
& \left|V_{c d}\right|=0.224 \pm 0.012
\end{aligned}
$$


Table 9: LQCD impact (in per cent) on the precision of CKM matrix elements. A charm factory data set of $3 / \mathrm{fb}$ and a B factory data set of $500 / \mathrm{fb}$ is assumed.

\begin{tabular}{lrrrrrr}
\hline & $V_{c d}$ & $V_{c s}$ & $V_{c b}$ & $V_{u b}$ & $V_{t d}$ & $V_{t s}$ \\
\hline 2004 & 7 & 11 & 4 & 15 & 36 & 39 \\
LQCD & 2 & 2 & 3 & 5 & 5 & 5 \\
\hline
\end{tabular}

Due to the large theoretical uncertainties in the CLEO-c numbers the extracted values of $V_{c s}$ and $V_{c d}$ should be considered as tests of LQCD. Nonetheless, they are the single most precise determinations of $V_{c s}$ and $V_{c d}$ to date. With $0.75 \mathrm{fb}^{-1}$ of data the CLEO-c precision is expected to be respectively:

$$
\begin{aligned}
& \left|V_{c s}\right|=\sqrt{0.8 \% \oplus \delta \Gamma / 2 \Gamma} \\
& \left|V_{c d}\right|=\sqrt{1.6 \% \oplus \delta \Gamma / 2 \Gamma}
\end{aligned}
$$

Where $\delta \Gamma / \Gamma$ is the uncertainty in the partial rate from theory. This in turn allows new unitarity tests of the CKM matrix. For example, the second row of the CKM matrix can be tested at the few \% level. With the current measurements I find:

$$
1-\left(\left|V_{c s}\right|^{2}+\left|V_{c d}\right|^{2}+\left|V_{c b}\right|^{2}\right)=0.037 \pm 0.181
$$

which is consistent with unitarity, with an uncertainty dominated by the LQCD charm semileptonic form factor magnitude The measurements also allow the first column of the CKM matrix to be tested with similar precision to the first row (which is currently the most stringent test of CKM unitarity); finally, the ratio of the long sides of the $u c$ unitarity triangle will be tested to a few percent.

Table 9 provides a summary of projections for the precision with which the CKM matrix elements will be determined if LQCD passes the charm factory tests in the $D$ system. In the tabulation the current precision of the CKM matrix elements is obtained by considering methods applicable to LQCD, for example the determination of $\left|V_{c b}\right|$ and $\left|V_{u b}\right|$ from inclusive decays and OPE is not included. The projections are made assuming $B$ factory data samples of $500 \mathrm{fb}^{-1}$ and improvement in the direct measurement of $\left|V_{t b}\right|$ expected from the Tevatron experiments 5 ). 


\subsection{The bottom line}

How can we be sure that if LQCD works for $D$ mesons it will work for $B$ mesons? Or, equivalently, is charm factory data sufficient to demonstrate that lattice systematic errors are under control? There are a number of reasons to answer this question in the affirmative. (1) There are two independent effective field theories: NRQCD and the Fermilab method. (2) The CLEOc, and later BESIII, data provide many independent tests in the $D$ system; leptonic decay rates, and semileptonic modes with rate and shape information. (3) The $B$ factory data provide additional independent cross checks such as $d \Gamma(B \rightarrow \pi \ell \nu) / d p_{\pi}$. (4) Unlike models, methods used for the $D / B$ system can be tested in heavy onia with measurements of masses, and mass splittings, $\Gamma_{e e}$ and electromagnetic transitions. (5) The main systematic errors limiting accuracy in the $D / B$ systems are: chiral extrapolations in mlight, perturbation theory, and finite lattice spacing. These are similar for charm and beauty quarks. In my opinion a combination of CLEO-c and BES III data in the $D$ systems and onia, plus information on the light quark hadron spectrum, can clearly establish whether or not lattice systematic errors are under control.

While this picture is encouraging, experimentalists also have concerns. The lattice technique is all encompassing but LQCD practitioners are very conservative about what can be calculated. For example when there was a hint that $\sin 2 \beta\left(\psi K_{S}^{0}\right) \neq \sin 2 \beta\left(\phi K_{S}^{0}\right)$, and when CP violation was observed in

$B \rightarrow K \pi$ (38) the lattice was not able to contribute. There is a pressing need to move beyond the limited set of easy to calculate quantities in the next few years: for example resonances such as $\rho, \phi$ and $K^{*}$ may be difficult to treat on the lattice, but they feature in many important $D$ semileptonic decays which will be well measured by the charm factories. There is also a need to be able to calculate for states near threshold such as $\psi(2 S)$ and $D_{s}(0)^{+}$, and hadronic weak decays in the $B$ and $D$ systems as well.

\section{New physics searches with charm}

In the early part of the 20th Century table top nuclear $\beta$ decay experiments conducted at the $\mathrm{MeV}$ mass scale probed the $W$ at the $100 \mathrm{GeV}$ mass scale. In an analogous way can we find violations of the Standard Model by studying low energy processes? The existence of multiple fermion generations appears to 
originate at very high mass scales and so can only be studied indirectly. Mixing, $\mathrm{CP}$ violation, and rare decays may investigate the new physics at these scales through intermediate particles entering loops. Why is charm a good place to look? In the charm sector, the SM contributions to these effects are small, in other words, a background free search for new physics is possible (see caveats below). Typically $D^{0}-\bar{D}^{0}$ mixing $\mathcal{O}\left(<10^{-2}\right)$, CP asymmetry $\mathcal{O}\left(<10^{-3}\right)$ and rare decays $\mathcal{O}\left(<10^{-6}\right)$. In addition, charm is a unique probe of the up-type quark sector (down quarks in the loop). The sensitivity of searches for new physics in charm depends on high statistics rather than high energy.

\subsection{Charm Mixing}

Mixing has been a fertile ground for discoveries. The neutral kaon mixing amplitude occurs at the same order as the kaon decay width $\propto\left|V_{u s}\right|^{2}$ and so the mixing rate is of order unity. The mixing rate, which vanishes in the SU(4) symmetry limit, was measured in 1958, was used to bound the charm quark mass, 16 years before the discovery of charm. The CP violating part of $K^{0} \bar{K}^{0}$ mixing, $\epsilon_{K}$, first measured in 1964 was a crucial clue that the top quark existed, thirty years before its discovery. In the $B^{0} \bar{B}^{0}$ system the top quark dominates the mixing amplitude, the $B$ decay width is Cabibbo suppressed $\propto\left|V_{c b}\right|^{2}$ and mixing is also Cabibbo suppressed $\propto\left|V_{t d}\right|^{2}$. The mixing rate is again of order unity, which was an early indication that $m_{\text {top }}$ was large. In $D^{0} \bar{D}^{0}$ mixing the amplitude is proportional to $\sin ^{2} \theta_{c} \sim 0.05$ but the decay width is not Cabibbo suppressed $\left(V_{c s} \sim 1\right)$. There is additional GIM suppression of order $\left(m_{s}^{2}-m_{d}^{2}\right) / m_{W}^{2}=0$ in the $\mathrm{SU}(3)$ limit, and so the rate for $D$ mixing in the SM is the product of Cabibbo suppression and an $\mathrm{SU}(3)$ breaking term, the latter being extremely difficult to estimate 39 )

$$
\text { mixing } \sim \sin ^{2} \theta_{c} \times[S U(3) \text { breaking }]^{2}
$$

In consequence, SM predictions span the range bounded by the experimental upper limit of $1 \%$ and the short distance box diagram rate of $\mathcal{O}\left(10^{-8}\right)$ 40) and the di-penguin rate $\mathcal{O}\left(10^{-10}\right)$ 41). New physics predictions span the same large range 42), implying that the observation of $D$ mixing alone is not a clear indication of new physics. However, the current experimental bounds $\mathcal{O}\left(10^{-2}\right)$ 43, 44, 45) already constrain new physics models. 
Table 10: Summary of measurements of $y_{C P}$

\begin{tabular}{|lll|}
\hline & \multicolumn{1}{c|}{$\%$} \\
\hline Belle & 2003 & $1.15 \pm 0.69 \pm 0.38$ \\
BABAR & 2003 & $0.8 \pm 0.4_{-0.4}^{+0.5}$ \\
CLEO & 2001 & $-1.1 \pm 2.5 \pm 1.4$ \\
Belle & 2001 & $-0.5 \pm 1.0_{-0.8}^{+0.7}$ \\
FOCUS & 2000 & $3.4 \pm 1.4 \pm 0.7$ \\
E791 & 1996 & $0.8 \pm 2.9 \pm 1.0$ \\
\hline
\end{tabular}

Neutral meson mixing is characterized by two dimensionless parameters

$$
x=\Delta M / \Gamma, y=\Delta \Gamma / 2 \Gamma
$$

where $\Delta m=m_{1}-m_{2}$ is the mass difference and $\Delta \Gamma=\Gamma_{1}-\Gamma_{2}$ is the width difference between the two neutral $D$ meson $C P$ eigenstates, and $\Gamma$ is the average width. If mixing occurs either $x$ or $y$ or both are non-zero.

The lifetime difference $y$ is constructed from the decays of a $D$ into physical states, and so it is expected to be dominated by SM contributions. In addition to the tiny SM contribution, the mass difference, $x$, is sensitive to new particles in the box diagram loop. Thus, new physics can significantly modify $x$, leading to $x>>y$. This signature for new physics is lost, however, if a relatively large $y$ of $\mathcal{O}(1 \%)$ is observed 46). As CP violating effects in mixing in the SM must involve the third quark generation, and since the bottom quark contribution to the box diagram is highly suppressed, $\propto V_{c b} V_{u b}^{*}$, the observation of $C P$ violating effects in $D$ mixing would be an unambiguous signal of new physics.

Mixing, and $C P$-violation in mixing, can be searched for in a variety of ways. Measurements of $y$ are summarized in Table 10 and are reviewed in 44) 43) 45). The world average is

$$
y_{C P}=(0.9 \pm 0.4) \%
$$

In the limit of $C P$-conservation $y_{C P}=y$. The $95 \%$ C.L. range of $y$ is the horizontal band in Figure 8

Searching for $D$ mixing in semileptonic decays is straightforward as there 
Table 11: Summary of searches for $D^{0}$ mixing with semileptonic decays. (Limits are $90 \%$ C.L.)

\begin{tabular}{|lll|}
\hline & $R_{M}$ U. L. $\times 10^{-3}$ \\
\hline Belle & 2005 & 1.0 \\
CLEO & 2005 & 7.8 \\
BABAR & 2004 & 4.2 \\
FOCUS & 2002 & 1.01 \\
E791 & 1996 & 5.0 \\
\hline
\end{tabular}

is an unambiguous signal that mixing has occurred:

$$
\begin{gathered}
D^{*+} \rightarrow D^{0} \pi_{\mathrm{tag}}^{+}, D^{0} \rightarrow K^{-} e^{+} \nu_{e} \text { unmixed } \\
D^{*+} \rightarrow D^{0} \pi_{\mathrm{tag}}^{+}, D^{0} \rightarrow \overline{D^{0}} \rightarrow K^{+} e^{-\overline{\nu_{e}}} \text { mixed }
\end{gathered}
$$

The flavor of the $D$ meson at birth is tagged by the sign of the pion from the $D^{*}$, the flavor at decay is tagged by the sign of the lepton. The time evolution of a neutral $D$ meson depends on the type of state into which it decays, and it is particularly straightforward for semileptonic final states.

$$
\begin{gathered}
\Gamma_{\text {unmix }} \propto e^{-t / \tau} \\
\Gamma_{\text {mix }} \cong e^{-t / \tau}(t / \tau)^{2} \frac{1}{4}\left(x^{2}+y^{2}\right)
\end{gathered}
$$

where $t$ is the proper time of the $D^{0}$ decay, and the approximation is valid in the limit of small mixing rates. The time integrated mixing rate relative to the unmixed rate is

$$
R_{\text {mix }}=\frac{1}{2}\left(x^{2}+y^{2}\right)
$$

The rate depends quadratically on $x$ and $y$ and does not provide a way to differentiate between them. Table 11$]$ is a compilation of results. The $95 \%$ CL limit on $R_{\text {mix }}$, a circular region centered on $(x=0, y=0)$ is displayed in Figure 8

Another way to search for $D$ mixing is in the hadronic decay $D^{0} \rightarrow K^{-} \pi^{+}$. This method is sensitive to a linear function of $x^{2}$ and $y$, and can differentiate between them. The most restrictive mixing constraints come from this mode. 
Table 12: Mixing searches using $D^{0} \rightarrow K^{+} \pi^{-}$. Comparison of the 95\% C.L. limits in per cent for the fit output parameters when $C P$ conservation is assumed in the fit. The FOCUS entries are one dimensional limits.

\begin{tabular}{|cccc|}
\hline & $R_{D}\left[\times 10^{-3}\right]$ & $y^{\prime}[\%]$ & $x^{\prime 2} / 2[\%]$ \\
\hline 2006 Belle 47) & $3.77 \pm 0.08 \pm 0.05$ & $(-2.8,2.1)$ & $<0.036$ \\
2005 FOCUS 48) & $4.29 \pm 0.63 \pm 0.28$ & $(-11.0,6.6)$ & $<0.385$ \\
2003 BABAR 49) & $3.57 \pm 0.22 \pm 0.27$ & $(-5.6,3.9)$ & $<0.11$ \\
2000 CLEO 50) & $3.32_{-0.65}^{+0.63} \pm 0.40$ & $(-5.8,1.0)$ & $<0.041$ \\
\hline
\end{tabular}

The unmixed signal is the Cabibbo favored (CF) $D^{0} \rightarrow K^{-} \pi^{+}$. The mixed signal is $D^{0} \rightarrow \bar{D}^{0} \rightarrow K^{+} \pi^{-}$but it has a background from doubly Cabibbo suppressed (DCS) decays $D^{0} \rightarrow K^{+} \pi^{-}$. Interference between the CF and DCS decays, which is linear in $y^{\prime}$, gives rise to the power of the method. The proper decay time distribution is fit to distinguish between DCS and the mixing signal. For $|x|,|y| \ll 1$ and negligible $C P$-violation, the decay time distribution for $D^{0} \rightarrow K^{+} \pi^{-}$is

$$
\frac{d N}{d t}=\left[R_{D}+\sqrt{R_{D}} y^{\prime} \Gamma t+1 / 4\left(x^{\prime 2}+y^{\prime 2}\right)(\Gamma t)^{2}\right] e^{-\Gamma t}
$$

where $R_{D}$ is the ratio of DCS to CF decay rates. In principle, there is a strong phase difference, $\delta_{K \pi}$, between the CF and DCS amplitudes which rotates $x$ and $y$ to $x^{\prime}$ and $y^{\prime}$. To search for $C P$-violation one determines $R_{D}, x^{\prime}$ and $y^{\prime}$ separately for $D^{0}$ and $\bar{D}^{0}$. Most recent analyses have been made both with and without requiring $C P$ conservation. Table 12 is a compilation of results. Figure 8 shows: (a) That the Belle recent analysis is an impressive step forward in sensitivity. (b) There remains no statistically significant evidence for $D$ meson mixing although the situation is becoming increasingly tantalizing.

At a charm factory as $\psi(3770) \rightarrow D \bar{D}$ and $C=-1$ quantum coherence guarantees that the mixing signature $D^{0} \rightarrow K^{-} \pi^{+}, \overline{D^{0}} \rightarrow D^{0} \rightarrow K^{-} \pi^{+}$, cannot be mimicked by one $D$ undergoing a DCS decay. Combining with semileptonic decays to increase sensitivity, a $0.75 / \mathrm{fb}(15 / \mathrm{fb})$ sample reaches $x<$ $1.7 \%(x<0.4 \%)$. A more sophisticated approach: The Quantum Correlated Analysis (TQCA) makes a combined fit to single and double flavor and $C P$ tag yields, which are a function of $\mathcal{B}_{i}, x^{2}, y, \delta_{i}$. TQCA is estimated to achieve a sensitivity for $0.75 / \mathrm{fb}(10 / \mathrm{fb}, 1,000 / \mathrm{fb})$ of $x<2.4 \%(x<1.3 \%, x<0.1 \%)$ and 


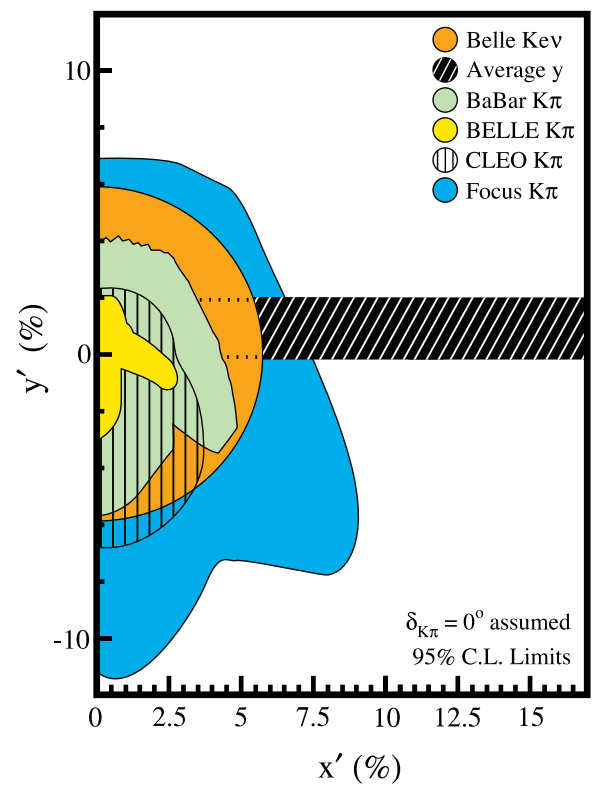

Figure 8: The status of searches for D meson mixing at the 95\% C.L. The semicircle $x^{2}+y^{2}$ is the most restrictive limit from semileptonic decays. For $D^{0} \rightarrow K^{+} \pi^{-}, x-y$ contours are shown separately for Belle, BABAR, CLEO, and FOCUS. For the former three, the limits allow for $C P$ violation in the decay amplitude, the mixing amplitude, and the interference between these two processes. To place $y \delta_{k \pi}=0$ is assumed. The world average allowed range for $y$ is the horizontal band. If $\delta_{k \pi} \neq 0$ the allowed $y$ region would rotate clockwise about the origin by an angle $\delta_{k \pi}$.

$y<1.2 \%(y<0.3 \%, y<0.03 \%)$. Purohit showed at this workshop that a B Factory with $10 /$ ab in a $D(t) \rightarrow K^{-} \pi^{+}$analysis reaches $x<1 \%$ and Nakada showed that at LHC-b in one year an analysis of $D(t) \rightarrow K^{0} \pi \pi$ reaches a sensitivity of $x<0.4 \%$.

\subsection{Measurement of the hadronic phase}

At the $\psi(3770)$ if a $D^{0}$ is observed to decay to a $C P$ eigenstate which is $C P$ even: then in the limit of $C P$ conservation, the state recoiling against the tag has a definite $C P$ as well and it must be of opposite sign, in this case $C P$ odd. Consider the situation where the second $D$ decays to a flavor mode:

$$
\sqrt{2} A\left(D_{C P \pm} \rightarrow K^{-} \pi^{+}\right)
$$




$$
=A\left(D^{0} \rightarrow K^{-} \pi^{+}\right) \pm A\left(\overline{D^{0}} \rightarrow K^{-} \pi^{+}\right)
$$

which defines two triangles from which $\cos \delta_{K \pi}$ can be determined. Determining $\delta_{K \pi}$ is necessary to rotate $x^{\prime}$ and $y^{\prime}$ measured in $D(t) \rightarrow K \pi^{-}$to $x$ and $y$. The method is limited by the number of $C P$ tags, but can be extended to many modes simultaneously in the TQCA where the sensitivity for $0.75 / \mathrm{fb}(10 / \mathrm{fb})$ is $\cos \delta_{K \pi} \pm 0.13( \pm 0.05)$.

\subsection{Charm contributions to $\phi_{3} / \Gamma$}

The phase of $V_{u b}, \phi_{3} / \Gamma$ can be determined by the interference between $b \rightarrow u$ and $b \rightarrow c$ decays where the $D$ decays to a $C P$ eigenstate or a flavor mode. In the first the $D$ mixing parameters are needed and in the second knowledge of $\cos \delta_{K \pi}$. Both methods require very large integrated luminosity. A third method, the Dalitz method, is currently the most accessible method experimentally. Here $B \rightarrow D K^{+}, D \rightarrow K_{S}^{0} \pi \pi$ With this approach the B factories have measured $\phi_{3}=68 \pm 14 \pm 13 \pm 11^{\circ}$ [11), and $\gamma=67 \pm 28 \pm 13 \pm 11$ [52), where the third uncertainty is from the $D$ decay model and can be reduced by analyzing $C P$ tagged Dalitz plots for $D \rightarrow K_{S}^{0} \pi \pi$ at charm factories. A study by Bondar 53) estimates statistical uncertainty on $\phi_{3} / \gamma$ at a B-Factory from $B \rightarrow D K^{+}$to be $\left( \pm 6^{\circ}\right.$ for $1 / \mathrm{ab}$ and $\pm 2^{\circ}$ for $10 / \mathrm{ab}$. The integrated luminosity needed to provide the number of $C P$ tagged $D \rightarrow K_{S}^{0} \pi \pi$ to match the statistical uncertainty from the super B factory for $\pm 6^{\circ}$ is $0.75 / \mathrm{fb}$ and $\pm 2^{\circ}$ for $10 / \mathrm{fb}$. The latter is a good match to the capabilities of BES III. CLEO-c sensitivity $(281 / \mathrm{pb})$ is consistent with Bondar's prediction

\subsection{Charm CP Violation}

Three types of $C P$ violation are possible. (1) $C P$ violation in the $D^{0}-\bar{D}^{0}$ mixing matrix. As $D$ mixing is very small, $C P$-violation in $D$ mixing, commonly parameterized by $A_{\mathrm{m}}$, is negligible both in the SM and many of its extensions. Experiments are not yet statistically sensitive to it, and so we will not consider it. (2) $C P$ violation in the interference between mixing and decay. It is time dependent, since mixing is involved but it is also small since $D$ mixing is suppressed. It is a good place to search for new physics, but experiment is only now becoming sensitive enough. (3) Direct $C P$ violation. This occurs when the absolute value of the $D$ decay amplitude to a final state $f$ is not equal to 
the $C P$-conjugate amplitude

For direct $C P$-violation to occur, two amplitudes with different weak phases and different strong phases must contribute to the decay process. The expression for the $C P$ asymmetry $A_{C P}$ is

$$
\begin{array}{r}
A_{\mathrm{CP}}=\frac{\Gamma\left(D^{0} \rightarrow f\right)-\Gamma\left(\overline{D^{0}} \rightarrow f\right)}{\Gamma\left(D^{0} \rightarrow f\right)+\Gamma\left(\bar{D}^{0} \rightarrow f\right)} \\
=\frac{2 \operatorname{Im} A_{1} A_{2} \sin \left(\delta_{1}-\delta_{2}\right)}{\left|A_{1}\right|^{2}+\left|A_{2}\right|^{2}+2 R e A_{1} A_{2}^{*} \cos \left(\delta_{1}-\delta_{2}\right)}
\end{array}
$$

where $A_{i}, \delta_{1}$ and $\delta_{2}$ are the moduli of the amplitudes, the weak phase difference and the strong phase differences, respectively.

In the $\mathrm{SM}$, direct $C P$ violation in the $D$ meson system occurs for singly Cabibbo suppressed decays such as $D^{0} \rightarrow \pi^{+} \pi^{-} / K^{+} K^{-} / K^{+} K^{-} \pi^{+}$, because for these decays there are several candidates for the second weak amplitude including penguin graphs, WA diagrams for $D_{s}^{+}$decays, and channels with a $K_{S}^{0}$ 54).

Predictions for $A_{C P}$ are difficult due to the unknown strong phase. In the SM $A_{C P}<10^{-3}$. New physics can produce $A_{C P} \approx 1 \%$. However, if an asymmetry at the $1 \%$ level was observed, one could not rule out a hadronic enhancement of the SM. Therefore it is necessary to analyze many channels to elucidate the source of $\mathrm{CP}$ violation. Selected measurements of $A_{\mathrm{CP}}$ are shown in Figure 9 Sensitivity approaches $1 \%$.

At the $\psi(3770) \mathrm{CP}$ violating asymmetries can be measured by searching for events with two $\mathrm{CP}$ odd or two $\mathrm{CP}$ even final states. For $D \rightarrow K^{+} K^{-}$ charm factory sensitivity for $(0.75 / \mathrm{fb}, 10 / \mathrm{fb}, 1,000 / \mathrm{fb})$ is $\left(A_{C P}<0.08,<\right.$ $4 \times 10^{-3},<6 \times 10^{-5}$ ) at $90 \%$ C.L. Nakada has shown that in one year at LHC-b the sensitivity is $A_{C P}<1.4 \times 10^{-4}$. An alternative is to search for $C P$ violation in $D \rightarrow f, D_{C P} \rightarrow$ flavor mode. The sensitivities at charm factories are $\left(A_{C P}<0.025,<6 \times 10^{-3},<7 \times 10^{-4}\right)$ at $90 \%$ C.L. respectively.

Alternative search strategies include Dalitz plot analyses that are particularly sensitive since they probe $C P$-violating phases in the amplitude rather than in the rate, are beginning to be attempted. These can be performed at charm threshold exploiting the quantum coherence and at higher energies. 


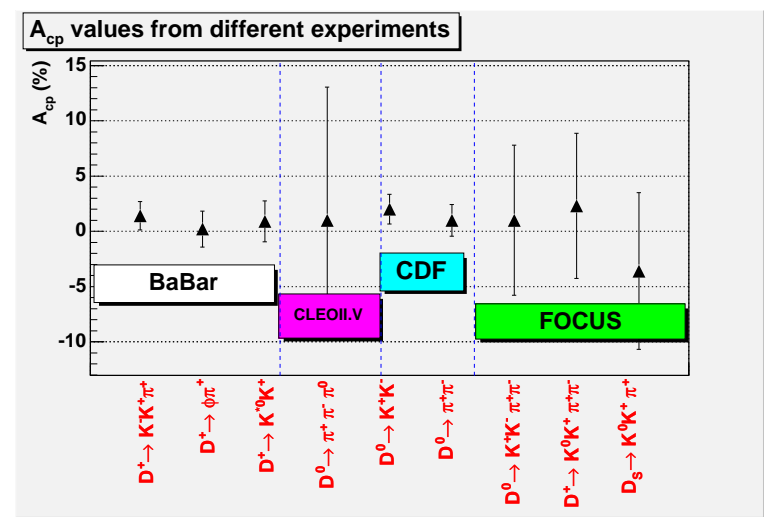

Figure 9: Selected searches for direct $C P$ violation in $D$ decay.

\subsection{Rare Decays}

In the SM flavor changing neutral currents are suppressed by the GIM mechanism. The dilepton decay proceeds by penguin annihilation or a box diagram. SM expected branching ratios are $\mathcal{B}\left(D^{0} \rightarrow e^{+} e^{-}\right) \sim 10^{-23}, \mathcal{B}\left(D^{0} \rightarrow\right.$ $\left.\mu^{+} \mu^{-}\right) \sim 3 \times 10^{-13}$. The lepton flavor violating mode $D^{0} \rightarrow e^{ \pm} \mu^{\mp}$ is strictly forbidden. New physics may enhance these processes. For example $R$-parity violating SUSY predicts $\mathcal{B}\left(D^{0} \rightarrow e^{+} e^{-}\right) \leq 10^{-10}, \mathcal{B}\left(D^{0} \rightarrow \mu^{+} \mu^{-}\right) \leq 10^{-6}$ and $D^{0} \rightarrow e^{ \pm} \mu^{\mp} \leq 10^{-6}$ [55). The result of a BABAR search which significantly improved upon previous upper limits $[56$, is shown in Figure 10 and Table 13 respectively. BES III will reach a sensitivity of few $\times 10^{-7}$ a super flavour factory at $10 \mathrm{GeV}$ with $50 /$ ab will achieve afew $\times 10^{-9}$ and if operated at the $\psi(3770)$ also afew $\times 10^{-9}$. However the $10 \mathrm{GeV}$ measurement is likely to be compromised by large backgrounds while the $\psi(3770)$ measurement will have little background and so the latter will be far superior, although still four orders of magnitude above the SM rate.

If new physics is present in rare $D$ decays it is likely to be more experimentally accessible in the modes $D \rightarrow X \ell^{+} \ell^{-}$. In the SM the $\mathcal{B}\left(D^{+} \rightarrow \pi e^{+} e^{-}\right)=$ $2.0 \times 10^{-6}$. In R-parity violating SUSY the integrated rate increases by only $20 \%$, however the differential dilepton mass distribution is significantly modified compared to the SM at low and high dilepton masses well away from the $\rho / \omega / \phi$ SM contributions. Several experiments have recently made searches, see 
Table 13: Selected recent searches for rare $D$ decays.

\begin{tabular}{|lll|}
\hline Mode & Upper Limit $\times 10^{-6}$ \\
\hline$e^{+} e^{-}$ & BABAR & 1.2 \\
$\mu^{+} \mu^{-}$ & BABAR & 1.3 \\
$e^{ \pm} \mu^{\mp}$ & BABAR & 0.81 \\
$\pi e^{+} e^{-}$ & CLEO-c & 7.4 \\
$K e^{+} e^{-}$ & BABAR & 3.6 \\
\hline
\end{tabular}

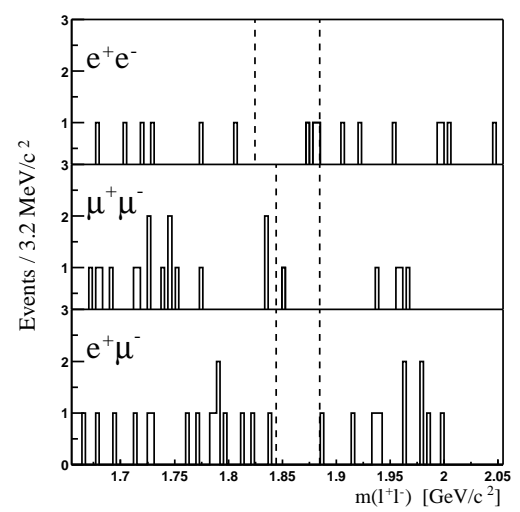

Figure 10: The di-lepton invariant mass distribution for $D^{0} \rightarrow e^{+} e^{-}, D^{0} \rightarrow$ $\mu^{+} \mu^{-}$and $D^{0} \rightarrow e^{ \pm} \mu^{\mp}$. The dashed lines indicate the signal mass region.

Table 13. If $D^{+} \rightarrow \pi e^{+} e^{-}$is at the SM level, only $1 \mathrm{evt} / \mathrm{fb}$ will be produced at the $\psi(3770)$ implying BESIII will observe 24 events (including a factor of two for $D^{+} \rightarrow \pi \mu^{+} \mu^{-}$.) While this sounds modest, just imagine if these event are clustered at low or high dilepton mass well away from SM contributions, it would be clear evidence for new physics!

In summary the experimental sensitivity for both $D^{0}$ and $D^{+}$rare decays is in the range $10^{-5}-10^{-6}$. For some modes, notably $D \rightarrow \pi^{+} \ell^{+} \ell^{-}$, measurements are beginning to confront models of new physics. In other cases, measurements are far above the SM prediction. The outlook for rare charm decays is promising. CDF, the B Factories, the charm factories, ATLAS/CMS and LHC-b will all contribute. For selected projections see 44). 


\section{SUMMARY}

New physics searches in the charm sector involving mixing, $C P$-violation and rare decays have become considerably more sensitive in the past several years, however, all results are null.

In charm's role as a natural testing ground for QCD techniques, there has been solid progress. Data at the $\psi(3770)$ from BESII and CLEO-c, and later BESIII, is finally producing a new era of precision absolute charm branching ratios. This is well-matched to developments in theory, especially the lattice, which has a goal to calculate to a few percent precision in the $D, B, \Upsilon$, and $\psi$ systems. CLEO-c, and later BES III, will provide few per cent precision tests of lattice calculations in the $D$ system and in heavy onia, which will quantify the accuracy for the application of LQCD to the $B$ system. If all goes to plan, BABAR, Belle, CDF, D0, CMS, ATLAS, and LHC-b data, in combination with LQCD will produce a few per cent determinations of $\left|V_{u b}\right|,\left|V_{c b}\right|,\left|V_{t d}\right|$, and $\left|V_{t s}\right|$ thereby maximizing the sensitivity of the flavor physics program to new physics beyond the SM this decade and aid understanding beyond the SM physics at the LHC in the coming decade.

\section{Acknowledgements}

I thank my colleagues on BABAR, Belle, BES II, CDF, CLEO, D0, FOCUS and LHC-b for many valuable discussions. Bo Xin is thanked for technical assistance. I am particularly grateful to Stefano Bianco for organizing a superb meeting in Frascati and for his patience while I completed this manuscript.

\section{References}

1. A.D. Sakharov, JETP Lett. 624 (1967).

2. C.T.H. Davies et al. Phys. Rev. Lett. 92022001 (2004).

3. CLEO-c/CESR-c Taskforces and CLEO Collaboration, Cornell LEPP Preprint CLNS 01/1742 (2001).

4. A decription of the BES III physics program and detector, and the design of the BEPCII accelerator may be found at the http://bes.ihep.ac.cn/conference/wksp04 
5. J. Swain and I. Taylor, Phys. Rev. D58 093006 (1998).

6. S. Eidelman et al. Phys Lett. B593 (2004) 1.

7. The Heavy Flavours Averaging Group (HFAG) summer 2005.

8. The PDG reports $\mathcal{B}\left(D \rightarrow \pi e^{+} \nu_{e}\right)$ with an error of $20 \%$, but the sole measurement, by the Mark III experiment, reports an error of $45 \%$.

9. G. Bellini, I.I. Bigi and P.J. Dornan, Phys. Rep. 2891 (1997).

10. BaBar Collaboration, B. Aubert et al. Phys. Rev. D 71091104 (2005).

11. MARK III Collaboration, R.M. Baltrusaitis et al., Phys. Rev. Lett. 56 2140 (1986).

12. MARK III Collaboration, J. Adler et al., Phys. Rev. Lett. 6089 (1988).

13. CLEO Collaboration, Q. He et al., Phys. Rev. Lett. 95121801 (2005).

14. ALEPH Collaboration R. Barate et al. Phys. Lett. B 405191 (1997).

15. The CLEO measurement of $\mathcal{B}\left(D^{0} \rightarrow K^{-} \pi^{+}\right)$is obtained by combining CLEO Collaboration, D.S. Akerib et al., Phys. Rev. Lett. 713070 (1993) and CLEO Collaboartion M. Artuso et al., Phys. Rev. Lett. 803193 (1998).

16. The CLEO measurement is obtined using 15) and CLEO Collaboration R. Balest et al., Phys. Rev. Lett. 722328 (1994).

17. MARK III Collaboratiion, J. Adler et al., Phys. Rev. Lett. 6089 (1988).

18. CLEO Collaboration, M. Artuso et al., arXiv:hep-ex/050857.

19. (MILC Collaboration) C. Aubin et al. hep-lat/0506030 (2005).

20. The BES II Collaboration M. Abilikim et al. Phys. Lett. B 610, 183 (2005).

21. CLEO Collaboration, G.S. Huang et al., Phys. Rev. Lett. 951818101 (2005). CLEO Collaboration T.E. Coan et al., Phys. Rev. Lett. 95181802 (2005).

22. CLEO Collaboration, G.S. Huang et al., Phys. Rev. Lett.94 011802 (2005). 
23. BES Collaboration, M. Ablikim et al. hep-ex/0410030 submitted to Phys. Lett. (2004).

24. BES Collaboration, M. Ablikim et al. Phys. Lett. B597 39 (2004).

25. J.D. Richman and P. R. Burchat Rev. Mod. Phys. 67893 (1995).

26. D. Becirevic and A.B. Kaidelov, Phys. Lett. B478, 417 (2000).

27. E691 Collaboration J.C. Anjos et al., Phys. Rev. Lett. 621587 (1989).

28. CLEO Collaboration, G. Crawford et al., Phys. Rev. D 44, 3394 (1991).

29. MARK III Collaboration, A. Bai et al., Phys. Rev. Lett. 66, 1011 (1991).

30. CLEO Collaboration, A. Bean et al., Phys. Lett. B 317, 647 (1993).

31. E687 Collaboration, P.L. Fabretti et al., Phys. Lett. B 317, 647 (1995).

32. FOCUS Collaboration, J.M. Link et al., Phys. Let. B697, 233 (2005).

33. Belle Collaboration, L. Widhalm et al., hep-ex/0604049.

34. BABAR Collaboration, J.D. Jackson talk at Charm2006 Beijing, China (2006).

35. C.Aubin et al., Phys. Rev. Lett. 94011601 (2005).

36. A. Khodjamirian et al., Phys. Rev. D62, 014002 (2000).

37. D. Melikhov and B. Stech, Phys. Rev. D 62, 014006 (2000).

38. BABAR Collaboration, B. Aubert et al., Phys. Rev. Lett. 93131801 (2004).

39. A.F. Falk, Y. Grossman, Z. Ligeti and A.A. Petrov, Phys. Rev. D 6505034 (2002); A.F. Falk, Y. Grossman, Z. Ligeti, Y, Nir and A.A. Petrov, Phys. Rev. D 69114021 (2004).

40. A. Datta and D. Kumbhakar, Z. Phys. C27, 515 (1985).

41. A.A. Petrov, Phys. Rev D56, 1685 (1997), hep-ph/9703335

42. A. A. Petrov hep-ph/0311371 
43. S. Bianco, F.L. Fabbri, D. Benson and I. Bigi, Riv. Nuovo Cim. 26N7-8, 1 (2003). hep-ex/0309021

44. G. Burdman and I. Shipsey, Ann. Rev. Nucl. Part. Sci., (2003). hep-ph/0310076

45. D. Asner $D^{0} \overline{D^{0}}$ Mixing Review to appear in PDG 2006.

46. S. Bergmann, Y. Grossmann, Z. Ligeti, Y. Nir, A. Petrov, Phys. Lett. B486, 418 (2000).

47. Belle Collaboration L. M. Zhang et al. arXiv:hep-ex/060124 submitted to Phys. Rev. Lett.

48. FOCUS Collaboration, J.M. Link et al. Phys. Lett. B 61823 (2005).

49. BABAR Collaboration, Aubert B, et al. Phys. Rev. Lett. 91171801 (2003).

50. CLEO Collaboration, R. Godang, et al. Phys. Rev. Lett. 845038 (2000).

51. Belle Collaboration Phys. Rev D 70072003 (2004).

52. BABAR Collaboration hep-ex/0507101

53. A. Bondar hep-ph/0510246

54. I.I. Bigi and A.I. Sanda, Phys. Lett. B171 320 (1986).

55. G. Burdman et al., Phys. Rev. D66 014009 (2002).

56. BABAR Collaboration, B. Aubert et al. Phys. Rev. Lett. 93101801 (2005). 